\title{
Channel capacity and performance evaluation of precoded MIMO-OFDM system with large-size constellation
}

\author{
Hussein A. Leftah ${ }^{1}$, Huda N. Alminshid ${ }^{2}$ \\ ${ }^{1}$ Basrah engineering technical college (BETC), Southern technical university (STU), Basrah, Iraq \\ ${ }^{2}$ Basrah technical institute, Southern technical university (STU), Basrah, Iraq
}

\begin{tabular}{l} 
Article Info \\
\hline Article history: \\
Received Jan 29, 2019 \\
Revised Mey 25, 2019 \\
Accepted Jun 25, 2019
\end{tabular}

\section{Keywords:}

Channel capacity

Multiple input-multiple output

Orthogonal Frequency

Division Multiplexing,

MMSE equalizer

Quadrature Amplitude

Modulation

\section{Corresponding Author:}

Hussein A. Leftah

Basrah engineering technical college (BETC), southern technical university (STU),

Basrah, +964 7729766659, Iraq.

Email: h.a.leftah@stu.edu.iq

\begin{abstract}
Multiple input-multiple output (MIMO) is a multipath diversity exploring approach which is emerged with orthogonal frequency division multiplexing (OFDM) to produce MIMO-OFDM that is widely used in wireless communications. This paper presents a discrete Hartley transform (DHT) precoded MIMO-OFDM system over multipath frequency-selective fading channel with large-size quadrature amplitude modulation (16-QAM, 64-QAM and 256-QAM). A mathematical models for the BER and channel capacity over mutlipath fading channels are also derived in this paper. Average Bit-error-rate (BER) and channel capacity of the presented system is considered and compared with that of the traditional MIMO-OFDM. Simulation results shows that the transmission performance and channel capacity of the proposed schemes is better than that of the traditional MIMO-OFDM without a precoder.
\end{abstract}

Copyright (c) 2019 Insitute of Advanced Engineeering and Science. All rights reserved.

\section{INTRODUCTION}

Increasing interest in wireless broadband communications and interactive media is fuelling up intensive research for higher-speed data rate transmission, bandwidth efficiency and bit-error-rate (BER) performance over multipath fading channels $[1,2]$. Owing to the popular characteristics of the orthogonal frequency division multiplexing (OFDM) in bandwidth efficiency, simple channel equalization and immunity to multipath fading channels, it has been considered for several wireless applications and standards [3, 4]. It has been adopted in IEEE $802.11 \mathrm{a}$,g,and n, IEEE 802.16a, and terrestrial digital video broadcasting (DVB-T) [5].

Multiple input-multiple output (MIMO) technology has received an exceptional consideration in the last decade. This is because its achievement in channel capacity and transmission reliability over wireless channels [6]. MIMO technology that is combined with the OFDM, commonly known as MIMO-OFDM, is widely considered in the literature[7, 8]. The MIMO-OFDM technology is a bright technique for considerable wireless applications as a bandwidth efficient and performance improved technology. However, the average BER of MIMO-OFDM systems could be affected by various channel impairment and nonlinear devices. Therefore, a new developed techniques are constantly being on demand for further improving the MIMO-OFDM performance.

Furthermore, a plethora of research has been presented supporting that the MIMO-OFDM can signinficantly explore the multipath diversity if a proper designed precoder is used [9-13], a precoder was designed at the transmitter based on the channel elements. The system throughput of this approach is low 
due to the feedback link that is used for the channel. It also required further complexity and it is hard to apply when the channel is fast fading. A unitary precoder with limited feedback was designed for spatial multiplexing MIMO-OFDM [12]. In [13], a channel independent unitary precoder was presented. The main drawback of this approach is its high complexity as it is required extra transforms at the transmitter and receiver. To solve this problem, we presented in our previous work [14] an Alamouti space time (ST-X-OFDM) based on a unitary orthogonal low complexity $\mathbf{X}$ tranform which is emegrging the effects of discrete Hartley transform and the effects of DFT together in single transform. However, the system in [14] does not include channel capacity, has not introduced a mathematical formula for the bit-error-rate (BER) performance and it was not generalized to MIMO with massive antennas.

To solve the diversity problem in MIMO-OFDM, a DHT precoded MIMO-OFDM with $N_{t}$ transmit antennas and $N_{r}$ receive antennas over multipath frequency-selective fading channel is presented in this paper with large-size qadrature amplitude modulation (16-QAM, 64-QAM and 256-QAM). We also present a mathematical model for the average BER and channel capacity of the DHT precoded MIMO-OFDM is also derived. The DHT precoded MIMO-OFDM system could not only reduce the transmitter complexity [14] but also enhance the channel capacity and exploit the multipath channels diversity and achieves noticeable signal-to-noise ratio (SNR) improvement in comparison to the classic MIMO-OFDM. The presented system also achieves better channel capacity than the conventional MIMO-OFDM system.

The remaining of this research paper is formulated as follows; system model and mathematical model for general $N_{t}$ and $N_{r}$ antenna diversity is presented in section 2. Numerical results and is shown in section 3. The conclusions are drawn in section 4 .

\section{SYSTEM MODEL}

The complex baseband systems' block diagram of MIMO system is depicted in Figure 1. In this work, $N_{t}$ transmit and $N_{r}$ receive antennas $\left(N_{t} \times N_{r}\right)$ MIMO system is considered in this paper. The input data is converted into M-array QAM symbols. These mapped symbols, $\mathbf{S}$, are assumed to be zero-mean with equal power distribution. The covariance matrix of the mapped data is given as

$$
\mathbf{C}_{S}=E\left[\mathbf{S S}^{*}\right]=E_{s} \mathbf{I}_{N}
$$

where $E_{s}$ is the symbol power and $\mathbf{I}_{N}$ is the identity matrix of $N$ dimensions. The DHT transform is then used to modulate these data symbols as

$$
\begin{aligned}
r_{n} & =\mathbf{H}_{n} \mathbf{S} \\
& =\sum_{m=0}^{N-1} h_{n, m} S_{m} .
\end{aligned}
$$

In (2b), $\mathbf{H}_{n}$ represents the $n_{t h}$ row of the DHT transform, and $h_{n, m}$ represents the $n_{t h}$ row, $m_{t h}$ column elements of $\mathbf{H}$. In matrix form, equation (2) is given as

$$
\mathbf{r}=\mathbf{H S}
$$

These precoded data symbols are then further modulated by the inverse FFT (IFFT) as

$$
\begin{aligned}
\mathbf{s}^{(b)} & =\mathbf{F}^{\mathbb{H}} \mathbf{r}^{(b)}, \\
\mathbf{s}^{(b)^{\dagger}} & =\mathbf{F}^{\mathbb{H}} \mathbf{r}^{(b)^{*}},
\end{aligned}
$$

where $\mathbf{s}^{(b)^{\dagger}}=\left[s_{1}^{(b)^{*}}, s_{2}^{(b)^{*}}, \cdots, s_{N-1}^{(b)}{ }^{*}, s_{0}^{(b)^{*}}\right]^{T}, \mathbf{F}$ is the discrete Fourier transform (DFT) and $(.)^{\mathbb{H}}$ represents the Hermitian, complex conjugate, operation. 


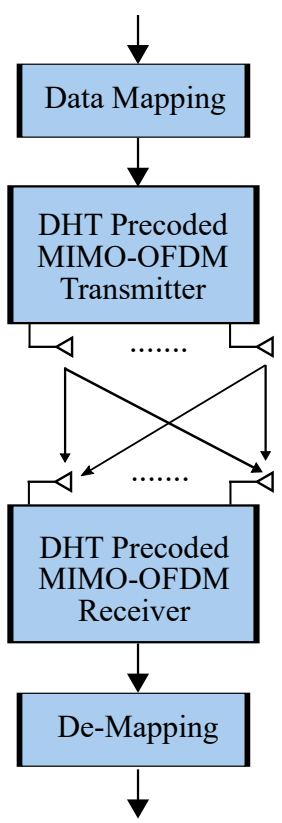

Figure 1. Schematic diagram of the presented scheme

The transmission diversity and performance improvement are increased as the number of antennas diversity increased. In the case of $N_{r}$ transmitter antennas, the frequency domain signal is expressed as [15]:

$$
\left[\begin{array}{ccccc}
\mathbf{r}^{(1)} & \mathbf{r}^{(2)} & \cdots & \mathbf{r}^{\left(N_{t}-1\right)} & \mathbf{r}^{\left(N_{t}\right)} \\
-\mathbf{r}^{(2)} & \mathbf{r}^{(1)} & \cdots & -\mathbf{r}^{\left(N_{t}\right)} & \mathbf{r}^{\left(N_{t}-1\right)} \\
\vdots & \vdots & \ddots & \vdots & \vdots \\
-\mathbf{r}^{\left(N_{t}-1\right)^{*}} & \mathbf{r}^{\left(N_{t}\right)^{*}} & \cdots & \mathbf{r}^{(1)^{*}} & -\mathbf{r}^{(2)^{*}} \\
-\mathbf{r}^{\left(N_{t}\right)^{*}} & -\mathbf{r}^{\left(N_{t}-1\right)^{*}} & \cdots & \mathbf{r}^{(2)^{*}} & \mathbf{r}^{(1)^{*}}
\end{array}\right]
$$

where the row represents the space diversity ( signal assigned to each antenna) whereas the column represents the time diversity ( signal assigned to the every next transmission).

The received frequency domain signal at the specific $n_{r_{t h}}$ antenna is expressed as

$$
\mathbf{Y}=\mathbf{G r}+\mathbf{\Omega},
$$

where $\mathbf{Y} \in \mathbb{C}^{N_{t} \times 1}, \mathbf{G} \in \mathbb{C}^{N_{t} \times N_{t}}, \mathbf{r} \in \mathbb{C}^{N_{t} \times 1}$ and $\boldsymbol{\Omega} \in \mathbb{C}^{N_{t} \times 1}$

In the above equation, the following definitions were used:

$$
\mathbf{r}=\left[\mathbf{r}^{(1)}, \mathbf{r}^{(2)}, \cdots, \mathbf{r}^{\left(N_{t}\right)}\right]^{T}
$$

and the channel matrix $\mathbf{G}$ is given as

$$
\mathbf{G}=\left[\begin{array}{ccccc}
\mathbf{g}^{\left(1, n_{r}\right)} & \mathbf{g}^{\left(2, n_{r}\right)} & \cdots & \mathbf{g}^{\left(N_{t}-1, n_{r}\right)} & \mathbf{g}^{\left(N_{t}, n_{r}\right)} \\
\mathbf{g}^{\left(2, n_{r}\right)} & -\mathbf{g}^{\left(1, n_{r}\right)} & \cdots & \mathbf{g}^{\left(N_{t}, n_{r}\right)} & -\mathbf{g}^{\left(N_{t}-1, n_{r}\right)} \\
\vdots & \vdots & \ddots & \vdots & \vdots \\
\mathbf{g}^{\left(N_{t}-1, n_{r}\right)^{*}} & -\mathbf{g}^{\left(N_{t}, n_{r}\right)^{*}} & \cdots & -\mathbf{g}^{\left(1, n_{r}\right)^{*}} & \mathbf{g}^{\left(2, n_{r}\right)^{*}} \\
\mathbf{g}^{\left(N_{t}, n_{r}\right)^{*}} & \mathbf{g}^{\left(N_{t}-1, n_{r}\right)^{*}} & \cdots & -\mathbf{g}^{\left(2, n_{r}\right)^{*}} & -\mathbf{g}^{\left(1, n_{r}\right)^{*}}
\end{array}\right]
$$

The detected signal is obtained by multiplying the signal in (6) by the complex conjugate (Hermitian) of the channel matrix as

$$
\tilde{\mathbf{Y}}=\mathbf{G}^{\mathbb{H}} \mathbf{Y} .
$$


Substitute (6) in (9) yields

$$
\tilde{\mathbf{Y}}=\mathbf{G}^{\mathbb{H}} \mathbf{G r}+\mathbf{G}^{\mathbb{H}} \boldsymbol{\Omega}
$$

In (10), the term $\left(\mathbf{G}^{\mathbb{H}} \mathbf{G}\right)$ is $N_{t} \times N_{t}$ diagonal matrix, $\boldsymbol{\Sigma}$, where the main diagonal is given as $\Sigma_{n}=\sum_{n_{t}=1}^{N_{t}}\left|g_{n}^{\left(n_{t}, n_{r}\right)}\right|^{2}$, $n=1,2, \ldots, N_{t}$. Hence, (10) could be rewritten as:

$$
\tilde{\mathbf{Y}}=\boldsymbol{\Sigma} \mathbf{r}+\mathbf{G}^{\mathbb{H}} \boldsymbol{\Omega}
$$

The MMSE equalizer is performed as

$$
\begin{aligned}
\hat{\mathbf{r}} & =\Delta \tilde{\mathbf{Y}} \\
& =\Delta\left(\boldsymbol{\Sigma} \mathbf{r}+\mathbf{G}^{\mathbb{H}} \boldsymbol{\Omega}\right) \\
& =\Delta \boldsymbol{\Sigma} \mathbf{r}+\Delta \mathbf{G}^{\mathbb{H}} \boldsymbol{\Omega},
\end{aligned}
$$

where $\boldsymbol{\Delta}$ is the MMSE equalizer which is defined as:

$$
\begin{aligned}
\boldsymbol{\Delta} & =\frac{E_{s}}{E_{s} \boldsymbol{\Sigma}+\sigma_{v}^{2} \mathbf{I}_{N_{t}}}, \\
& =\frac{\gamma_{s}}{\mathbf{I}_{N_{t}}+\gamma_{s} \boldsymbol{\Sigma}} .
\end{aligned}
$$

The DHT transform is then used to process the equalized signal to produce the QAM-mapped symbols as

$$
\begin{aligned}
\mathbf{q} & =\mathbf{H} \hat{\mathbf{r}} \\
& =\mathbf{H} \boldsymbol{\Delta} \boldsymbol{\Sigma} \mathbf{H}^{\mathbb{H}} \mathbf{S}+\mathbf{H} \boldsymbol{\Delta} \mathbf{G}^{\mathbb{H}} \boldsymbol{\Omega} \\
& =\mathbf{H} \frac{\gamma_{s} \boldsymbol{\Sigma}}{\mathbf{I}_{N_{t}}+\gamma_{s} \boldsymbol{\Sigma}} \mathbf{H}^{\mathbb{H}} \mathbf{S}+\mathbf{H} \frac{\gamma_{s}}{\mathbf{I}_{N_{t}}+\gamma_{s} \boldsymbol{\Sigma}} \mathbf{G}^{\mathbb{H}} \boldsymbol{\Omega} .
\end{aligned}
$$

The signal power, $\mathcal{P}_{s}=E\left[|\mathbf{q}|^{2}\right]$, is as given as

$$
\begin{aligned}
\mathcal{P}_{s} & =|\mathbf{H}|^{2} \frac{E_{s} \gamma_{s}^{2} \boldsymbol{\Sigma}^{2}}{\left(\mathbf{I}_{N_{t}}+\gamma_{s} \boldsymbol{\Sigma}\right)^{2}}+|\mathbf{H}|^{2} \frac{E_{s} \gamma_{s} \boldsymbol{\Sigma}}{\left(\mathbf{I}_{N_{t}}+\gamma_{s} \boldsymbol{\Sigma}\right)^{2}} \\
& =|\mathbf{H}|^{2} \frac{E_{s} \gamma_{s} \boldsymbol{\Sigma}}{\mathbf{I}_{N_{t}}+\gamma_{s} \boldsymbol{\Sigma}}
\end{aligned}
$$

The noise signal, $\boldsymbol{\varepsilon}=\mathbf{q}-\mathbf{S}$, is given as:

$$
\begin{aligned}
\boldsymbol{\varepsilon} & =\left(\mathbf{H} \frac{\gamma_{s} \boldsymbol{\Sigma}}{\mathbf{I}_{N_{t}}+\gamma_{s} \boldsymbol{\Sigma}} \mathbf{H}^{\mathbb{H}}-\mathbf{I}_{N_{t}}\right) \mathbf{S}+\mathbf{H} \frac{\gamma_{s}}{\mathbf{I}_{N_{t}}+\gamma_{s} \boldsymbol{\Sigma}} \mathbf{G}^{\mathbb{H}} \boldsymbol{\Omega} \\
& =\frac{-\mathbf{H S}}{\mathbf{I}_{N_{t}}+\gamma_{s} \boldsymbol{\Sigma}}+\mathbf{H} \frac{\gamma_{s}}{\mathbf{I}_{N_{t}}+\gamma_{s} \boldsymbol{\Sigma}} \mathbf{G}^{\mathbb{H}} \boldsymbol{\Omega} .
\end{aligned}
$$

The noise power, $\mathcal{P}_{n}=E\left[|\varepsilon|^{2}\right]$, is as given in

$$
\begin{aligned}
\mathcal{P}_{n} & =|\mathbf{H}|^{2} \frac{E_{s}}{\left(\mathbf{I}_{N_{t}}+\gamma_{s} \boldsymbol{\Sigma}\right)^{2}}+|\mathbf{H}|^{2} \frac{E_{s} \boldsymbol{\Sigma}}{\left(\mathbf{I}_{N_{t}}+\gamma_{s} \boldsymbol{\Sigma}\right)^{2}} \\
& =|\mathbf{H}|^{2} \frac{E_{s}}{\mathbf{I}_{N_{t}}+\gamma_{s} \boldsymbol{\Sigma}}
\end{aligned}
$$

Then the average SNR of the detected signal is given as

$$
S N R=\frac{\mathcal{P}_{s}}{\mathcal{P}_{n}}
$$


The channel capacity measured in (bits/s/Hz) is then calculated as

$$
C=\log _{10}\left(\mathbf{I}_{N}+S N R\right) .
$$

The BER, for the M-QAM signalling, is given as [16]

$$
P_{e}^{M-Q A M}=\frac{4-2^{\frac{4-\log _{2} M}{2}}}{\log _{2} M} Q\left(\sqrt{\frac{3 \mathcal{P}_{s}}{\mathcal{P}_{n}(M-1)}}\right) .
$$

where $m$ is number of bits in each data symbol.

\section{SIMULATION RESULTS}

In this section we present numerical results to assess the transmission efficiency of the precoded MIMO-OFDM and compared it with the classic MIMO-OFDM system. In this simulation N=1024 are used. For simulation simplicity we used antenna diversity as $2 \times 1$ and $2 \times 2$, however, the results could be generalized for any number of transmitter antennas $\left(N_{t}\right)$ and receiver antennas $\left(N_{r}\right)$. The multipath Rayleigh fading channel is represented by the pedestrian type B international telecommunication union (ITU) channel, its impulse response is obtained by Nyquist sampling and shown in Figure 2. This is a standard channel that is widely used in wireless communication applications. It is clear from Figure 2 that this channel has six statistically independent taps with non light of sight with maximum spread delay 43 taps.

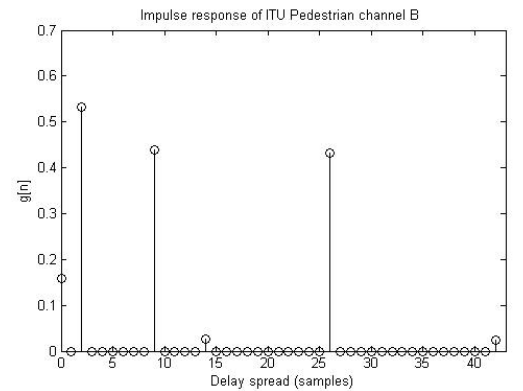

Figure 2. ITU pedestrian B Channel impulse response

\subsection{Channel Capacity}

Channel capacity is considered as outstanding metrics to measure the ability of wireless broadband communications to handle maximum data rate with reliable transmission. Higher channel capacity means that the telecommunication system can handle more applications and data transmission within a certain channel bandwidth. Therefore, system with high channel capacity, that is able to meet the market and customers requirements, is highly on demand. In this section, Computer simulation is used to investigate the capacity of the DHT-precoded MIMO-OFDM over Rayleigh fading ITU channel. Figure 3 shows the capacity in bits/s/Hz of the DHT-precoded MIMO-OFDM in comparison with the classic MIMO-OFDM over ITU pedestrian B multipath channel.

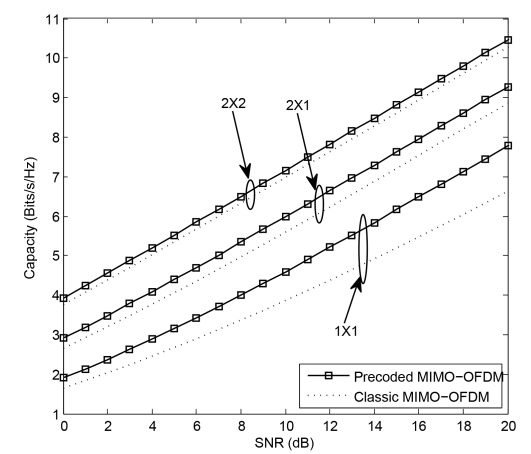

Figure 3. Channel capacity of the DHT precoded MIMO-OFDM system normalized per unit bandwidth 
It is obvious from Figure 3 that, for different antenna diversity, the capacity of the scheme increases as the antenna diversity increased. It is also clear that the channel capacity per unit bandwidth of the presented MIMO-OFDM is higher than the channel capacity of the classic MIMO-OFDM for all the antenna number were used $(1 \times 1,2 \times 1$ and $2 \times 2)$.

\subsection{BER Performance}

This section presents the transmission performance of DHT-precoded MIMO-OFDM and compares it with the classic MIMO-OFDM that is not using a precoder in its structure, over ITU pedestrian B multipath channel for large-size constellation (16-QAM, 64-QAM and 256-QAM data signalling. The simulation is run for two types of antenna diversity $(2 \times 1$ as in Figure 4 and $2 \times 2$ as in Figure 5).

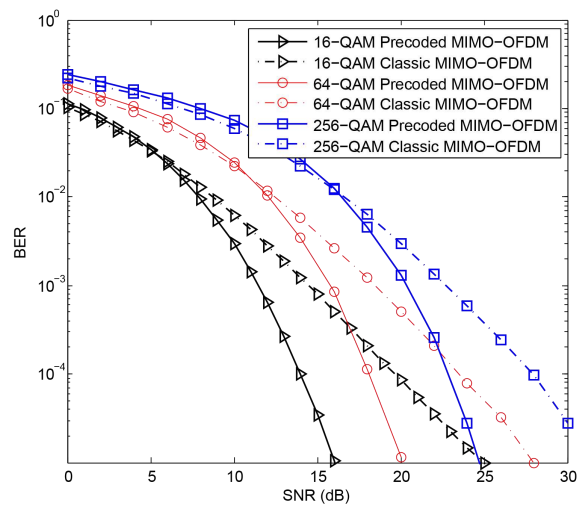

Figure 4. Average BER of DHT precoded MIMO-OFDM and classic MIMO-OFDM for 2X1 antenna diversity and 16-QAM, 64-QAM and 256-QAM data mapping

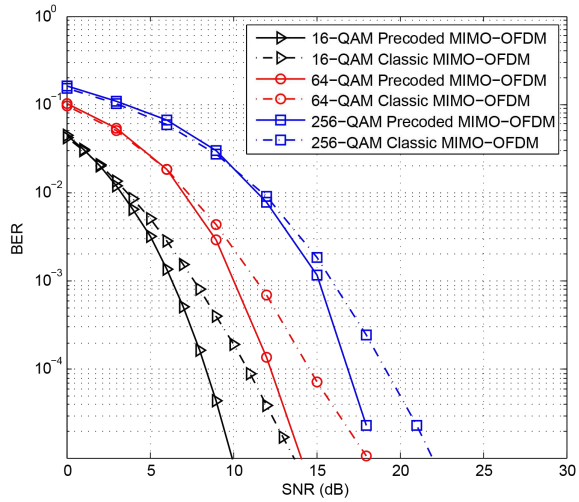

Figure 5. Average BER of DHT-precoded MIMO-OFDM and classic-OFDM for 2X1 antenna diversity and 16-QAM, 64-QAM and 256-QAM data mapping

For the case of $(2 \times 1)$, Figure 4 shows that at $10^{-5}$ BER, the precoded MIMO system gained about $8 \mathrm{~dB}$ SNR in comparison to classic MIMO scheme for all 16-QAM, 64-QAM and 256-QAM data mapping. This confirms that the DHT precoding MIMO-OFDM can explore the channel diversity. Figure 5 shows the average BER of the DHT precoded MIMO-OFDM in comparison to the classic MIMO-OFDM for 16-QAM, 64-QAM and 256-QAM data mapping. It is obvious from Figure 5 that the SNR gain due to the use of DHT as a precoder reduced from $8 \mathrm{~dB}$ in the case of $2 \times 1$ to $3 \mathrm{~dB}$ in the case of $2 \times 2$ diversity.

The reason why this SNR gain is reduced as the antenna diversity increased can be attributed the reason that the advantage of DHT precoded MIMO-OFDM over the classic MIMO-OFDM is on exploring the frequency diversity. Thus, for exploring the advantages of the DHT-precoded MIMO-OFDM, the channel type is a key factor. Therefore the antenna diversity contribute in reducing the effects of multipath channels and 
this is the reason why the SNR gain is lower as the antennas diversity increases. This is because the channel impairment on the transmitted signals is reduced as the number of antennas increased.

\section{CONCLUSION}

The transmission efficiency and channel capacity of DHT precoded MIMO-OFDM has been evaluated in this paper for large-size constellation and compared with that of the classic MIMO-OFDM scheme. Mathametical models for the channel capacity and average BER of the DHT-precoded MIMO-OFDM over multipath fading channels were also derived in this paper. Two types of antenna diversity $(2 \times 1$ and $2 \times 2$ were used. It has been shown that using DHT transform as a precoder in MIMO-OFDM over mutlipath fading channels can achieve valuable SNR gain and increase the channel capacity. This furnishing a promising system for transmission over severe multipath fading channel with large channel capacity which enables more data transmission within a certain bandwidth.

\section{REFERENCES}

[1] H. A. Leftah and S. Boussakta, "Efficient modulation scheme for OFDM system with ZP and MMSE equalizer," 2013 IEEE International Conference on Communications (ICC), Budapest, pp. 4703-4707, 2013.

[2] C. Han and Y. Chen, "Propagation Modeling for Wireless Communications in the Terahertz Band," in IEEE Communications Magazine, vol. 56, no. 6, pp. 96-101, June 2018.

[3] H. A. Leftah and S. Boussakta, "Novel OFDM Based on C-Transform for Improving Multipath Transmission," in IEEE Transactions on Signal Processing, vol. 62, no. 23, pp. 6158-6170, Dec.1, 2014.

[4] Z. Na, et al, "Modeling and Throughput Analysis of an ADO-OFDM Based Relay-Assisted VLC System for 5G Networks," in IEEE Access, vol. 6, pp. 17586-17594, 2018.

[5] A. Panajotovi, et al. "Adaptive Uniform Channel Decomposition in MU-MIMO-OFDM: Application to IEEE 802.11ac," in IEEE Transactions on Wireless Communications, vol. 14, no. 5, pp. 2896-2910, May 2015.

[6] S. P. Alex and L. M. A. Jalloul, "Performance Evaluation of MIMO in IEEE802.16e/WiMAX," IEEE Journal of selected Topics in Signal Processing, vol. 2, pp. 181-190, 2008.

[7] Y. G. Li, et al., "MIMO-OFDM for wireless communications: signal detection with enhanced channel estimation," in IEEE Transactions on Communications, vol. 50, no. 9, pp. 1471-1477, Sep 2002.

[8] A. Sakaushi, et al., "Performance evaluations of software-defined acoustic MIMO-OFDM transmission," 2018 IEEE Wireless Communications and Networking Conference (WCNC), Barcelona, Spain, pp. 1-6, 2018.

[9] D. Palomar, et al., Joint Tx-Rx beamforming design for multicarrier MIMO channels: a unified framework for convex optimization," IEEE Trans. Signal Process., vol. 51, no. 9, pp. 2381 2401, Sep. 2003.

[10] A. Punchihewa, et al., "Linear precoding for orthogonal space-time block coded MIMO-OFDM cognitive radio", IEEE Trans. Commun., vol. 59, no. 3, pp. 767779, Mar. 2011.

[11] T. Pande, et al., "Reduced Feedback MIMO-OFDM Precoding and Antenna Selection," in IEEE Transactions on Signal Processing, vol. 55, no. 5, pp. 2284-2293, May 2007.

[12] C. Jihoon, et al., "Interpolation based unitary precoding for spatial multiplexing MIMO-OFDM with limited feedback," in IEEE Global Telecommunications Conference (GLOBECOM), pp. 214 218, 2004.

[13] C. Yuan-Hwui and P. See-May, "Unitary precoders for ST-OFDM systems using Alamouti STBC," IEEE Transactions on circuits and Systems I: Regular Papers, vol. 55, pp. 2860 2869, 2008.

[14] H. A. Leftah, S. Boussakta and S. Ikki, "Enhanced Alamouti space-time block-coding transmission based on a developed OFDM system," 2014 9th International Symposium on Communication Systems, Networks and Digital Sign (CSNDSP), Manchester, pp. 591-595, 2014.

[15] A.B. Gershman and N.D. Sidiropoulos, "Space-Time Processing for MIMO Communications", John Wiley, 2005.

[16] J.G. Proakis and M. Salehi, "Digital Communications", McGraw-Hill Companies, Inc, 1221 Avenue of the Americas, New York, 2008. 